OPEN ACCESS

Edited by:

Ross Bailie,

University of Sydney, Australia

Reviewed by:

Marisa Theresa Gilles,

Western Australian Center for Rural

Health (WACRH), Australia

Jan Fizzell,

New South Wales Department of

Health, Australia

*Correspondence:

Sophie L. YorkWilliams

sophie.yorkwilliams@colorado.edu

Specialty section:

This article was submitted to

Public Health Policy,

a section of the journal

Frontiers in Public Health

Received: 14 December 2018

Accepted: 08 April 2019

Published: 30 April 2019

Citation:

YorkWilliams SL, Gust CJ, Mueller R, Bidwell LC, Hutchison KE, Gillman AS and Bryan AD (2019) The New

Runner's High? Examining Relationships Between Cannabis Use and Exercise Behavior in States With

Legalized Cannabis.

Front. Public Health 7:99.

doi: 10.3389/fpubh.2019.00099

\section{The New Runner's High? Examining Relationships Between Cannabis Use and Exercise Behavior in States With Legalized Cannabis}

\author{
Sophie L. YorkWilliams ${ }^{1 *}$, Charleen J. Gust ${ }^{1}$, Raeghan Mueller ${ }^{1}$, L. Cinnamon Bidwell ${ }^{2}$, \\ Kent E. Hutchison ${ }^{1}$, Arielle S. Gillman ${ }^{1}$ and Angela D. Bryan ${ }^{1}$ \\ ${ }^{1}$ Department of Psychology \& Neuroscience, University of Colorado Boulder, Boulder, CO, United States, ${ }^{2}$ Institute of \\ Cognitive Science, University of Colorado Boulder, Boulder, CO, United States
}

Scientific literature examining cannabis use in the context of health behaviors, such as exercise engagement, is extremely sparse and has yielded inconsistent findings. This issue is becoming increasingly relevant as cannabis legalization continues, a situation that has been associated with increased initiation of use among adults, and increased potency of available products in legalized states. Physical activity is among the most important health behaviors, but many Americans do not meet minimum exercise recommendations for healthy living. Common issues surrounding low exercise rates include inadequate enjoyment of and motivation to exercise, and poor recovery from exercise. It is unclear whether cannabis use shortly before and/or after exercise impacts these issues, and whether this co-use affects exercise performance. The present online survey study examines attitudes and behaviors regarding cannabis use with exercise among adult cannabis users living in states with full legal access $(N=605)$. Results indicated that the majority (81.7\%) of participants endorsed using cannabis concurrently with exercise, and those who did tended to be younger and more likely to be males ( $p<0.0005$ for both). Even after controlling for these differences, co-users reported engaging in more minutes of aerobic and anaerobic exercise per week $p<0.01$ and $p<0.05$, respectively). In addition, the majority of participants who endorsed using cannabis shortly before/after exercise reported that doing so enhances their enjoyment of and recovery from exercise, and approximately half reported that it increases their motivation to exercise. This study represents an important step in clarifying cannabis use with exercise among adult users in states with legal cannabis markets, and provides guidance for future research directions.

Keywords: cannabis, marijuana, exercise, cannabis legalization, health

\section{INTRODUCTION}

As evidenced by the increasing legalization of both medical and recreational cannabis in the United States (1), public acceptance of cannabis use is growing. Despite this, there is a relative lack of literature exploring the public health implications of cannabis consumption. One health behavior that may be impacted by increased cannabis use is exercise. The benefits of regular exercise are 
far-reaching and include the prevention or improvement of some cancers, cardiovascular disease, diabetes, depression, Alzheimer's disease, and memory loss (2-4). As a reflection of these benefits, the American College of Sports Medicine (ACSM) recommends that adults participate in at least $150 \mathrm{~min}$ of moderate- to vigorous-intensity exercise each week (5). Unfortunately, selfreport data indicate that fewer than $50 \%$ of US adults meet these minimum recommendations (6). These low rates of exercise participation are indicative of a significant and urgent health concern. Given popular stereotypes that cannabis use is associated with a lack of motivation and, indeed, extreme sedentary behavior (c.f., "couch lock"), increased cannabis use could worsen the sedentary lifestyle epidemic that the US is currently facing. On the other hand, cannabis use is banned by the World Anti-Doping Agency because of its potential to improve sports performance, and numerous reports in the popular press suggest that endurance athletes utilize cannabis in their training [e.g., Dreier (7)]. Such conflicting ideas about the relationship between cannabis use and physical activity necessitate further investigation into how cannabis use may affect factors associated with regular physical activity participation.

The sparse empirical literature base that speaks to the association between cannabis and health emphasizes potential negative and harmful effects associated with use, such as psychosis, impaired driving, and sleep disturbances (8-10). There is also an established relationship between cannabis use and increased caloric intake (11), which is especially worrisome given the current obesity epidemic; the Centers for Disease Control and Prevention reported in 2017 that nearly $40 \%$ of the US population is obese (12). However, recent results from representative national surveys suggest a lower prevalence of obesity in cannabis users than in non-users (13). Further, crosssectional data from the National Center for Health Statistics indicate positive correlations with cannabis use and measures of metabolic health, such as lower levels of fasting insulin, smaller waist circumference, and reduced prevalence of type II diabetes (14). There is also evidence to suggest that cannabis use is associated with a greater likelihood of meeting the aforementioned exercise guidelines (15), possibly due to positive influences on motivation or reductions in inflammation and pain during and after exercise (16).

Common barriers that prevent individuals from the initiation or continuation of a regular exercise regimen include (1) improper recovery after exercise (17), (2) lack of motivation (18), and (3) low enjoyment of exercise (19). Cannabis might interact with these barriers in contradictory ways, both positively (e.g., by promoting recovery from or enjoyment of exercise) and negatively (e.g., through decreases in motivation). Gillman et al. (16) address this notion in a review paper commenting on the biological and neurocognitive mechanisms by which cannabis use might impact three potential areas of influence: the effect of cannabis on exercise performance, motivation for exercise, and recovery from exercise. For example, Gillman et al. (16) report that few empirical studies have examined the relationship between cannabis use and exercise performance, and those that have are limited in their external validity due to the use of uncommon forms of cannabis for consumption (e.g., cannabis cigarettes containing unusually low-THC strains). Further, while anecdotal evidence suggests that cannabis reduces motivation for exercise, several neurobiological mechanisms connected to the endocannabinoid system argue against this; rather, there is evidence to suggest that cannabis may have beneficial effects on exercise motivation (16). Finally, while there is evidence that cannabis reduces pain and inflammation in humans (20), other research suggests the use of anti-inflammatory agents, such as cannabinoids, might actually interfere with proper recovery from exercise (16). These limitations and inconsistencies point to a clear need for additional investigations of the effects of cannabis use on health behaviors generally and on exercise in particular (16). Such research is particularly pressing in states with full legal cannabis access, as cannabis use is on the rise in these states and may include broader populations than have been represented in research conducted when cannabis use was an illegal behavior (21).

The present study aimed to address research questions set out by Gillman et al.'s review, and is the first to collect empirical data on attitudes and behaviors regarding cannabis use and exercise among current cannabis users living in states with full legal access. In particular, we were interested in examining differences between users who consume cannabis shortly before or after exercise (co-use), compared to those who do not. Building on Gillman et al. (16), we were also interested in attitudes regarding the impact of cannabis use before or after exercise on exercise performance, enjoyment, motivation, and recovery. Data were gathered using a web-based survey advertised to users in states with legal recreational cannabis. Since endorsement of the use of cannabis concurrent with exercise inherently increases the likelihood of exercise engagement (i.e., a cannabis user who does not exercise cannot use cannabis concurrent with exercise), we hypothesized that co-users would report more exercise behavior than non-co-users. Based on data supporting the anti-inflammatory and analgesic effects of cannabis (16), we hypothesized that users of cannabis concurrent with exercise would report that it aided in recovery. Finally, given conflicting findings regarding performance and motivation, and little to no data on enjoyment, we considered tests of these associations to be exploratory.

\section{METHODS}

\section{Procedures}

A voluntary, anonymous online "Cannabis and Health" survey was advertised on Facebook and targeted individuals aged 21 and older living in California, Colorado, Nevada, Oregon, and Washington and who "liked" pages related to cannabis use (e.g., Cannabis Culture Magazine). In Colorado, the survey was also advertised through medical cannabis card registration clinics and dispensaries in the Boulder-Denver area. The survey was hosted on Qualtrics.com and data were collected from January 2017 to July 2018. Survey details are described in another recent publication (22). Participants were asked demographic questions, along with health, exercise, and substance use questions. They were free to skip questions, other than acknowledgment of consent and that they were 21 years of age or older. Inclusion 
criteria for the current study were as follows: (1) reported living in a state with legal recreational cannabis, and (2) responded to the cannabis and exercise questions (details below).

In the initial iteration of the survey, we asked, "Do you ever use cannabis within 1 hour before and/or 4 hours after you engage in exercise?" This version was completed by 260 (43.0\%) of the present study participants. Subsequently, in an effort to delineate those who use cannabis either before or after exercise, the survey was altered and the remaining participants $(n=345 ; 57.0 \%$ of present study) were asked (1) "Do you ever use cannabis within 1 hour before you engage in exercise?" and (2) "Do you ever use cannabis within 4 hours after you engage in exercise?" Participants who answered "yes" to the single question in the first version, or "yes" to either question in the latter version, were categorized as individuals who use cannabis concurrent with exercise for the full-sample analyses.

Participants who endorsed cannabis use concurrent with exercise were given the following instructions. "The following statements are examples of why you might use cannabis before or after exercise. Please indicate how much you agree with each statement." They were presented with cannabis-exercise attitude statements (listed below) and asked to indicate their opinion of each on a 7 -point likert scale, with the anchors of $1=$ "strongly disagree," $4=$ "neither agree nor disagree," and $7=$ "strongly agree." Statements started with "I believe that" and ended with (1). cannabis enhances my exercise performance; (2). cannabis increases my enjoyment of exercise; (3). I am more motivated to engage in exercise when I use cannabis; (4). cannabis makes it easier to recover from my exercise experience (e.g., reduces inflammation, joint pain, etc.).

\section{Analyses}

RStudio software was utilized in all analyses. We first computed basic demographic information to describe the study sample, and calculated frequencies of concurrent exercise-cannabis use endorsement in the study. Group differences between those who endorsed cannabis use concurrent with exercise and those who did not were examined with $\chi^{2}$ tests or $t$-tests as appropriate, for age, gender, ethnicity, body mass index (BMI), state of residency, and frequency of cannabis use. Next, we used $t$ tests to examine differences between co-using and non-co-using groups on minutes of aerobic and anaerobic exercise per week. When group differences arose, we verified that they were not explained by demographic differences, by including demographic variables that were different between groups as covariates in linear regression models.

Next, we used two methods to examine cannabis-exercise attitudes among participants who endorsed co-use. First we examined attitudes numerically, by averaging scores for each of the 4 attitudes. We did this in the full co-using sample, and then examined whether average attitudes differed by type of co-use endorsement (cannabis use only before, only after, or both before and after) using between-subjects ANOVAs. This temporal specificity was only available in the 2-question version of the study, so participants from the 1-question version were included in the model as one group, and those from the 2question version were grouped by type of co-use endorsement.
Second, in the full sample of concurrent users, we categorized interpretations of these attitudes by percentage of participants who agreed or disagreed with each statement. A rating between 1 and 3 was categorized as "disagree," 4 as "neutral," and 5-7 as "agree."

\section{RESULTS}

\section{Study Demographics}

Among the 620 participants that completed either version of the survey and reported residency in a state with legal cannabis, 605 respondents provided answers to the cannabis-exercise questions and were included in the present study. Mean age was 37.5 (SD $=15.7 ; \min =21 ; \max =78)$, and $281(46.6 \%)$ were female. State of residency reports were: 419 (69.3\%) in Colorado, 99 (16.4\%) in California, 38 (6.3\%) in Oregon, $36(6.0 \%)$ in Washington, and $13(2.1 \%)$ in Nevada. The majority $(78.3 \%)$ of participants were white non-Hispanic, followed by $10.6 \%$ Hispanic, $4.0 \%$ Native American, 3.8\% black or African American, 1.5\% Asian, 0.05\% Pacific Islander, and $1.3 \%$ declined to answer.

\section{Cannabis Use Before/After Exercise}

In the full sample, 494 participants (81.7\%) endorsed using cannabis before and/or after exercise and 111 (18.3\%) did not. Among the sample from the first version of the survey, who answered the single cannabis-exercise question $(n=260), 79.6 \%$ endorsed using cannabis before and/or after exercise $(n=207)$, and $20.4 \%$ reported that they did not $(n=53)$. Among the 345 participants in the 2-question sample, 287 (83.3\%) endorsed at least one of the two cannabis-exercise questions. Specifically, 237 (68.9\%) endorsed using cannabis within $1 \mathrm{~h}$ before exercise, while $282(82.0 \%)$ endorsed using cannabis within $4 \mathrm{~h}$ after exercise, and $58(16.8 \%)$ reported that they did not use cannabis within $1 \mathrm{~h}$ before nor within $4 \mathrm{~h}$ after engaging in exercise. Interestingly, 232 of the 3452 -question sample participants endorsed using cannabis both before and after exercise $(67.2 \%)$, while $50(14.5 \%)$ endorsed only using after exercise, and only 5 (1.5\%) endorsed only using before exercise. Thus, most cannabis users who use cannabis concurrent with exercise report doing so both before and after exercise.

\section{Demographic and Lifestyle Differences Between Co-users and Non-co-users}

Bivariate relationships suggested that participants who used cannabis with exercise were younger, more likely to be male, and of lower BMI than those who did not use cannabis with exercise. Average age of participants who used cannabis with exercise was $36.3(\mathrm{SD}=14.9)$, while the average age of cannabis users who did not was 43.1 (SD $=18.0 ; p<0.0005 ; d=$ -0.39). Females comprised only $42.8 \%$ of participants who endorsed using cannabis with exercise, compared to $63.1 \%$ of those who did not $p<0.0005)$. Average reported BMI of cousing participants was $25.6(\mathrm{SD}=5.61)$, while the average was $27.0(\mathrm{SD}=6.0 ; p<0.05 ; d=-0.24)$ among those who did not use cannabis with exercise. However, these group differences in reported BMI were not present after age and gender, which were different between co-users and non-co-users, were accounted for. 
Participant race/ethnicity and state of residency did not differ between exercise groups.

Co-users endorsed using cannabis flower 5.5 days per week on average $(\mathrm{SD}=2.5 ; \min =0 ; \max =7)$, while non-cousers endorsed using 3.2 days $(\mathrm{SD}=3.1 ; \min =0$; $\max =7$; $p<0.0001 ; d=0.76)$. Co-users endorsed using high-potency cannabis concentrate 2.5 days per week on average $(\mathrm{SD}=2.9$; $\min =0 ; \max =7$ ), while non-co-users endorsed using 0.6 days $(\mathrm{SD}=1.6 ; \min =0 ; \max =7 ; p<0.0001 ; d=1.0)$. Co-users endorsed using cannabis edibles 1.3 days per week on average (SD $=2.0 ; \min =0 ; \max =7$ ), while non-co-users endorsed using 1.6 days $(\mathrm{SD}=2.4 ; \min =0 ; \max =7 ; n s)$. Even after controlling for age and gender, co-users used cannabis flower on 2.4 more days $(p<0.0001)$ and cannabis concentrates 1.8 more days $(p<$ $0.0001)$ than non-co-users on average.

\section{Differences in Exercise Behaviors Between Co-users and Non-co-users}

Co-using participants reported $159.7 \mathrm{~min}$ per week of aerobic exercise on average $(S D=154.3$; $\min =0$; $\max =600$ ), while non-co-users reported an average of $103.5 \mathrm{~min}(\mathrm{SD}=113.6$; $\mathrm{min}$ $=0 ; \max =510 ; p<0.0001 ; d=0.45)$. Using linear regression to control for potential demographic confounds of age and gender, use of cannabis with exercise was still associated with 43.4 more min of weekly aerobic exercise on average $(p<0.01)$. Consistent with this finding, $40.1 \%$ of cannabis users who used with exercise met or exceeded American College of Sports Medicine's recommendations of a minimum of $150 \mathrm{~min}$ of aerobic exercise per week (5), compared to only $28.7 \%$ of cannabis users who did not endorse using with exercise $(p=0.036)$. This discrepancy was not limited to aerobic activity. Cannabis users who used cannabis during exercise also reported an average of 37.4 more minutes of anaerobic exercise $($ mean $=101.6 ; \mathrm{SD}=129.1 ; \min =0 ; \max$ $=600$ ) than cannabis users who did not use during exercise (mean $=64.2 ; \mathrm{SD}=104.3 ; \min =0 ; \max =600 ; p<0.005 ; d=$ $0.33)$. After controlling for the demographic variables that were different between groups (age and gender), cannabis use during exercise was still associated with 30.2 more minutes of reported anaerobic exercise $(p=0.033)$.

\section{Attitudes About Cannabis Use Before/After Exercise}

Mean scores on the 1-7 scales for each cannabis-exercise attitude statement among all participants who endorsed co-use were as follows: performance was $4.4(\mathrm{SD}=1.6$; median $=4)$,

TABLE 1 | Attitudes on how use of cannabis with exercise impacts exercise performance, enjoyment, motivation, and recovery, among all participants who endorsed co-use $(N=494)$.

\begin{tabular}{lccc}
\hline & Mean & SD & Median \\
\hline Performance & 4.4 & 1.6 & 4 \\
Enjoyment & 5.3 & 1.6 & 6 \\
Motivation & 4.7 & 1.8 & 5 \\
Recovery & 5.7 & 1.5 & 6 \\
\hline
\end{tabular}

Rating options range from 1 ("strongly disagree) to 7 ("strongly agree"). enjoyment was 5.3 ( $\mathrm{SD}=1.6$; median $=6$ ), motivation was $4.7(\mathrm{SD}=1.8$; median $=5)$, and recovery was $5.7(\mathrm{SD}=1.5$; median $=6$ ). With a score of 4 equating to neither agreement nor disagreement, and 7 equating to strong agreement, results indicate that average attitudes, particularly toward enjoyment and recovery, were above neutral when the full co-using sample was combined (Table 1). Of note, more respondents endorsed strong agreement than strong disagreement with the attitude statements (i.e., there were more answers of 6 and 7 than 1 and 2). As such, the median results may represent participant attitudes better than the means and standard deviations. To add nuance to these attitudes, we wanted to examine whether they differed across participants who endorsed cannabis use only before, only after, or both before and after exercise. This level of detail was only available in the 2-question version of the study, so participants from the 1-question version were treated as one group. Due to the low number of survey participants who endorsed cannabis use only before exercise in the second version of the survey ( $n$ $=5$ ), they were not included in the ANOVA models. Thus, the 3 groups in the model were: those in the second survey who endorsed cannabis use both before and after exercise, those in the second survey who endorsed cannabis use only after exercise, and those in the first version of the survey who endorsed our single-question regarding cannabis use before or after exercise. Average scores for each attitude statement by group are detailed in Table 2. We found differences in subjective experience of those who reported cannabis use only after exercise, compared to those who used both before and after, and those who participated in the combined single-question survey version $(p<0.0001$ for all attitudes). This difference was most prominent for enjoyment, motivation, and performance.

In order to characterize these attitudes more broadly in all participants who endorsed use before and/or after exercise, we transformed these numeric ratings into categories, grouping all ratings below 4 ("neither agree nor disagree") as "disagree," and all ratings above 4 as "agree." Figure 1 provides a visual representation of these attitudes data. Among participants who endorsed using cannabis before and/or after exercise $(n=494)$, the majority $(70.7 \%)$ agreed or agreed strongly that cannabis increases enjoyment of exercise, $19.3 \%$ were neutral, and $10.0 \%$ disagreed or disagreed strongly. The majority (77.6\%) also agreed or agreed strongly that cannabis enhances recovery from exercise, while $16.3 \%$ were neutral and $6.1 \%$ disagreed or disagreed strongly. In contrast, just over half $(51.8 \%)$ agreed or agreed strongly that cannabis increases motivation to exercise, $26.5 \%$ were neutral, and $21.6 \%$ disagreed or disagreed strongly. Finally, a minority (37.5\%) agreed or strongly agreed that cannabis enhances exercise performance, while almost half (46.0\%) were neutral and $16.5 \%$ disagreed or disagreed strongly.

\section{DISCUSSION}

The current study aimed to characterize differences between adult cannabis users who consume cannabis shortly before and/or after exercising and those who do not, in the context of legal cannabis markets in the United States. Combining 


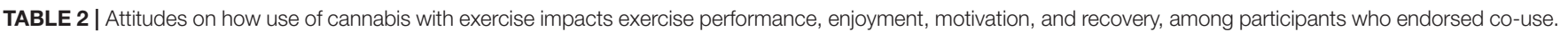

\begin{tabular}{|c|c|c|c|c|c|c|c|c|c|c|}
\hline \multirow[b]{2}{*}{ Version } & \multirow[b]{2}{*}{ Group } & \multirow[b]{2}{*}{$\mathbf{n}$} & \multicolumn{2}{|c|}{ Performance } & \multicolumn{2}{|c|}{ Enjoyment } & \multicolumn{2}{|c|}{ Motivation } & \multicolumn{2}{|c|}{ Recovery } \\
\hline & & & Mean & SD & Mean & SD & Mean & SD & Mean & SD \\
\hline \multirow[t]{2}{*}{ 2-question } & Before \& after & 230 & 4.7 & 1.5 & 5.5 & 1.4 & 5.0 & 1.7 & 5.8 & 1.3 \\
\hline & Only after & 50 & $2.8^{\star \star \star}$ & 1.3 & $3.2^{\star \star \star}$ & 1.6 & $2.9^{\star \star \star}$ & 1.5 & $5.2^{\star \star \star}$ & 1.8 \\
\hline 1-question & Co-users & 206 & 4.4 & 1.5 & 5.5 & 1.6 & 4.7 & 1.8 & 5.6 & 1.5 \\
\hline
\end{tabular}

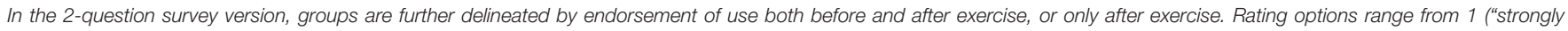
disagree) to 7 ("strongly agree"). " Group mean comparison $p<0.0001$.

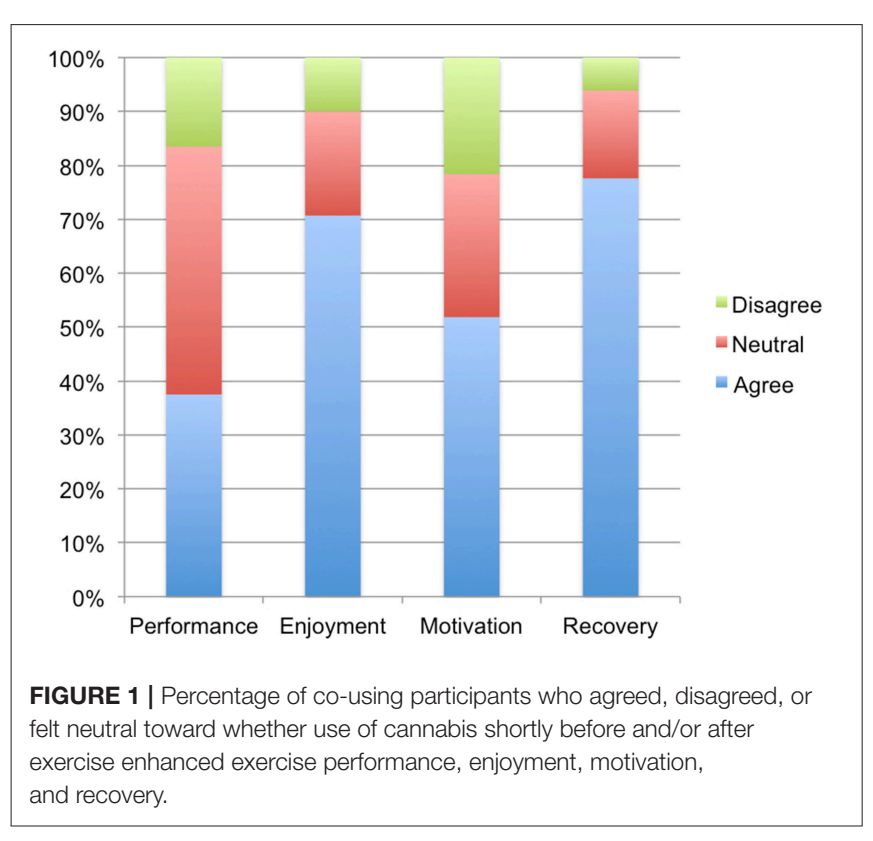

across survey versions of cannabis users, we found that the majority of our sample did endorse using cannabis concurrently with exercise. These participants were younger on average, and included a greater proportion of males than those who do not (both $p<0.0005)$. In line with our hypotheses, they also reported more minutes of aerobic and anaerobic exercise per week $(p$ $<0.01$ and $p<0.05$, respectively), even after accounting for potential demographic confounds of age and gender. Concurrent users reported consuming cannabis flower and concentrates more often than the non-co-using group (both $p<0.0001$, even after controlling for age and gender), but edible use did not differ between groups. Reasons for these correlations can only be speculated upon. It may be that more frequent cannabis users are more likely to engage in co-use simply by virtue of them using so often, rather than reasons related to exercise performance, enjoyment, motivation, or recovery.

Additionally, the present study sought to take initial steps in understanding possible mechanisms through which cannabis could influence exercise behavior, by examining attitudes toward how cannabis co-use influences the exercise experience. We found that the majority of participants who endorsed using cannabis concurrently with exercise reported that doing so at least somewhat enhances recovery from and enjoyment of exercise, while approximately half reported that it at least somewhat increases motivation, and a minority reported that it enhances performance. These findings supported our hypothesis that co-users may be co-using because they believe it contributes to recovery after exercise. The findings also suggest that co-use may facilitate enjoyment of exercise, and (for a subset of co-users) motivation to exercise. Given that these are recognized barriers to exercise (17-19), it is possible that cannabis might actually serve as a benefit to exercise engagement. Finally, the attitudes toward co-use and performance in our participants seem to concur with studies suggesting that cannabis use does not enhance exercise performance for most users (16).

To our knowledge, this is the first study to survey attitudes and behavior regarding the use of cannabis before and after exercise, and to examine differences between cannabis users who engage in co-use, compared to those who do not. Given both the spreading legalization of cannabis and the low rates of physical activity in the US, it behooves public health officials to understand the potential effects-both beneficial and harmfulof cannabis use on exercise behaviors (16). Cannabis use has become more common in the past decade, especially among adults in states with legal recreational cannabis (21). As legal risks associated with cannabis use decrease, it is plausible that a broader range of individuals will be initiating use. Thus, the present study's focus on legal market populations is timely, and results may help to anticipate the future landscape of other states, as legalization progresses.

Our results suggest that prior findings of cannabis users being more likely to meet official exercise recommendations (15) may be at least partly associated with perceived impacts of cannabis co-use on enjoyment, reductions in pain and inflammation during and after exercise, and to a lesser extent motivation. Furthermore, participants who use cannabis before and/or after exercise reported that they exercised more, and had positive attitudes about co-use on exercise, which implies cannabis may be a useful tool for exercise among some users. In other words, sedentary cannabis users, particularly those who attribute low physical activity to concerns about recovery, motivation, or enjoyment, may benefit from co-use, provided that they select low-risk exercise options that do not compromise safety during intoxication. However, since the present study did not query potential negative consequences of co-use, any recommendations are premature. For instance, the $16.5 \%$ of co-users who reportedly disagreed that co-use enhanced performance may exercise at a lower intensity when they use cannabis shortly beforehand. Similarly, the $6.1 \%$ who disagreed that co-use aided in recovery may have experienced injury from 
exercising while intoxicated. Clearly, more nuanced investigation is needed. Given the diversity of cannabis users in legal states (22), co-use may have a net-positive impact on some, and a netnegative impact for others. Potency and amount of cannabis used, type and context of exercise, and individual health status likely impact whether co-use is a safe and beneficial option.

Limitations of the current study include its cross-sectional, anonymous, and self-report nature, as well as recruitment issues, particularly that of cannabis users without a non-using comparison group. It is possible that recruitment through social media captured respondents who are invested in promoting cannabis, leading to results that are skewed favorably toward exercise. However, it is also possible that recruitment through medical cannabis card registration clinics might have captured respondents who are less physically able, thus leading to results skewed against exercise. It is also critical to recognize that there is substantial overlap between states that have legalized cannabis and states that have higher levels of physical activity. For whatever historical, political, or economic reasons, it is the case that states with legal access to cannabis are, without exception, more physically active than the national average (23). Without a non-using comparison group, we are unable to rule out potential selection bias; that is, it might be that non-users living in such highly active states are even more active than cannabis users. In addition, our findings about exercise behaviors and attitudes regarding cannabis co-use with exercise may not be representative of states with different cannabis legality and may not translate to future behaviors and attitudes if and when states with lower rates of physical activity legalize cannabis. However, given the positive associations that co-users reportedly experience between cannabis use and exercise enjoyment and recovery, there may indeed even be a stronger correlation between using cannabis with exercise and exercise behaviors in less active states. This will be a fascinating research question as more states move toward legalization. It is important to note that the current study represents a first attempt to elucidate this complex issue, and that there are currently no grounds for suggestion that non-users should initiate cannabis use to increase their physical activity participation.

As discussed above, future research is needed to refine and expand upon this foundational study. For instance, querying both users and non-users in legal states would clarify the extent to which cannabis use impacts exercise behaviors in these regions. For example, it could be that the non-cannabis users in these highly physically active states are even more active than cannabis users. We are unable to directly test this question with our survey data. Additionally, while the current study illuminates attitudes regarding co-use, it would be useful to explore how

\section{REFERENCES}

1. Cerdá M, Wall M, Feng T, Keyes KM, Sarvet A, Schulenberg J, et al. Association of state recreational marijuana laws with adolescent marijuana use HHS. JAMA Pediatr. (2017) 171:142-9. doi: 10.1001/jamapediatrics.2016.3624

2. Watson K, Baar K. mTOR and the health benefits of exercise. Semin Cell Dev Biol. (2014) 36:130-9. doi: 10.1016/j.semcdb.2014.08.013 often cannabis is used specifically to achieve exercise goals and in what contexts (e.g., specific forms of exercise and during group vs. individual exercise). It is particularly important to explore potential negative outcomes of cannabis co-use with exercise in future research, especially regarding accident or injury as a result of intoxication. Additionally, a longitudinal observational study design could be used to examine how changes in cannabinoid content and product potencies influence exercise behavior. Since we are unable to randomly assign sedentary individuals to use cannabis (or not) and subsequently observe exercise behavior, and because demographic differences can impact exercise engagement, propensity score matching could be used to estimate the effect cannabis legalization has on exercise behavior by equating co-users and non-co-users on influential covariates. Lastly, a useful tool to examine the influence of cannabis on exercise engagement at the population level would be to implement an interrupted time-series study design where exercise behavior is surveyed in a state before and after cannabis legalization.

In summary, these data suggest that many cannabis users in states with legal cannabis access use in conjunction with exercise, and that most who do so believe it increases enjoyment of, recovery from, and to some extent the motivation to engage in exercise. As these factors positively correlate with exercise behavior (24), using cannabis with exercise may play a beneficial role in the health of cannabis users. Recommendations around cannabis use with exercise are premature until aforementioned research questions have been addressed, including independent verification of self-reported behaviors and negative consequences of co-use.

\section{ETHICS STATEMENT}

This study was carried out in accordance with the recommendations of the University of Colorado Boulder's Institutional Review Board. Informed consent was given by all participants in accordance with the Declaration of Helsinki and all procedures were approved by the University of Colorado Boulder's Institutional Review Board.

\section{AUTHOR CONTRIBUTIONS}

$\mathrm{KH}$, LB, SY, and RM contributed to the design and implementation of the research. SY managed and analyzed the data. SY, AB, RM, and $\mathrm{LB}$ contributed to the interpretation of the results. SY, RM, and CG wrote the manuscript. SY, AB, CG, and LB edited the manuscript. All authors provided critical feedback and helped shape the manuscript. 
6. Schoenborn CA, Adams PF, Peregoy JA. Health behaviors of adults: United States, 2008-2010. Vital Health Stat 10. (2013) 257:1-184.

7. Dreier F. The debate over running while high. Wall Str J Online. (2015)

8. Asbridge M, Hayden JA, Cartwright JL. Acute cannabis consumption and motor vehicle collision risk: systematic review of observational studies and meta-analysis. BMJ. (2012) 344:e536. doi: 10.1136/bmj.e536

9. Garcia AN, Salloum IM. Polysomnographic sleep disturbances in nicotine, caffeine, alcohol, cocaine, opioid, and cannabis use: a focused review. Am J Addict. (2015) 24:590-8. doi: 10.1111/ajad.12291

10. Marconi A, Di Forti M, Lewis CM, Murray RM, Vassos E. Meta-analysis of the association between the level of cannabis use and risk of psychosis. Schizophr Bull. (2016) 42:1262-9. doi: 10.1093/schbul/sbw003

11. Rodondi N, Pletcher MJ, Liu K, Hulley SB, Sidney S. Marijuana use, diet, body mass index, and cardiovascular risk factors (from the CARDIA Study). Am J Cardiol. (2006) 98:478-84. doi: 10.1016/j.amjcard.2006.03.024

12. Hales CM, Carroll MD, Fryar CD, Ogden CL. Prevalence of obesity among adults and youth: United States, 2015-2016. Natl Cent Health Stat Data Br. (2017) 1-8.

13. Le Strat Y, Le Foll B. Obesity and cannabis use: results from 2 representative national surveys. Am J Epidemiol. (2011) 174:929-33. doi: 10.1093/ aje/kwr200

14. Penner EA, Buettner H, Mittleman MA. The impact of marijuana use on glucose, insulin, and insulin resistance among US adults. Am J Med. (2013) 126:583-9. doi: 10.1016/j.amjmed.2013.03.002

15. Vidot DC, Bispo JB, Hlaing WM, Prado G, Messiah SE. Moderate and vigorous physical activity patterns among marijuana users: results from the 2007-2014 National Health and Nutrition Examination Surveys. Drug Alcohol Depend. (2017) 178:43-8. doi: 10.1016/j.drugalcdep.2017.05.004

16. Gillman AS, Hutchison KE, Bryan AD. Cannabis and exercise science: a commentary on existing studies and suggestions for future directions. Sport Med. (2015) 45:1357-63. doi: 10.1007/s40279015-0362-3

17. Laux P, Krumm B, Diers M, Flor H. Recovery-stress balance and injury risk in professional football players: a prospective study. J Sports Sci. (2015) 33:2140-8. doi: 10.1080/02640414.2015.1064538
18. Nicholson S, Sniehotta FF, van Wijck F, Greig CA, Johnston M, McMurdo MET, et al. A systematic review of perceived barriers and motivators to physical activity after stroke. Int J Stroke. (2013) 8:357-64. doi: 10.1111/j.1747-4949.2012.00880.x

19. Zenko Z, Ekkekakis P, Ariely D. Can you have your vigorous exercise and enjoy it too? Ramping intensity down increases postexercise, remembered, and forecasted pleasure. J Sport Exerc Psychol. (2016) 38:149-59. doi: 10.1123/jsep.2015-0286

20. Kozela E, Juknat A, Kaushansky N, Rimmerman N, Ben-Nun A, Vogel Z. Cannabinoids decrease the inflammatory autoimmune phenotype. $J$ Neuroimmune Pharmacol. (2013) 8:1265-76. doi: 10.1007/s11481-013-9493-1

21. Williams AR, Santaella-tenorio J, Mauro CM, Levin FR, Martins SS. Loose regulation of medical marijuana programs associated with higher rates of adult marijuana use but not cannabis use disorder. Addiction. (2017) 112:1985-91. doi: 10.1111/add.13904

22. Bidwell LC, YorkWilliams SL, Mueller R, Bryan AD, Hutchison KE. Exploring cannabis concentrates on the legal market: user profiles, product strength, and health-related outcomes. Addict Behav Rep. (2018) 8:102-6. doi: 10.1016/j.abrep.2018.08.004

23. Warren M, Beck S, Rayburn J. The State of Obesity 2018. (2018). Available online at: www.tfah.org

24. Ingledew DK, Markland D. The role of motives in exercise participation. Psychol Health. (2008) 23:807-28. doi: 10.1080/08870440701405704

Conflict of Interest Statement: The authors declare that the research was conducted in the absence of any commercial or financial relationships that could be construed as a potential conflict of interest.

Copyright (c) 2019 YorkWilliams, Gust, Mueller, Bidwell, Hutchison, Gillman and Bryan. This is an open-access article distributed under the terms of the Creative Commons Attribution License (CC BY). The use, distribution or reproduction in other forums is permitted, provided the original author(s) and the copyright owner(s) are credited and that the original publication in this journal is cited, in accordance with accepted academic practice. No use, distribution or reproduction is permitted which does not comply with these terms. 